\title{
Partially conserved axial vector current and applications
}

R. Horsley ${ }^{a}$, S. Kazmin ${ }^{b}$, Y. Nakamura ${ }^{c}$, H. Perlt ${ }^{* b}$, P. E. L. Rakow ${ }^{d}$, G. Schierholz ${ }^{e}$ A. Schiller ${ }^{b}$ and J. M. Zanotti ${ }^{f}$

${ }^{a}$ School of Physics and Astronomy, University of Edinburgh, Edinburgh EH9 3FD, UK

${ }^{b}$ Institut für Theoretische Physik, Universität Leipzig, PF 100 920, D-04009 Leipzig, Germany

${ }^{c}$ RIKEN Advanced Institute for Computational Science, Kobe, Hyogo 650-0047, Japan

${ }^{d}$ Theoretical Physics Division, Department of Mathematical Sciences, University of Liverpool, Liverpool L69 3BX, UK

${ }^{e}$ Deutsches Elektronen-Synchrotron DESY, 22603 Hamburg, Germany

${ }^{f}$ CSSM, Department of Physics, University of Adelaide, Adelaide SA 5005, Australia

E-mail: perlteitp.uni-leipzig.de

We investigate implications of the use of the point-split axial vector current derived from a Wilson like fermionic action. We compute the corresponding renormalization factor nonperturbatively for one beta value. The axial charge $\mathrm{gA}$ calculated from this nonlocal current is found to be nearer to the physical value than computed with the local axial vector current - computed both on the same lattice with the same action.

34th annual International Symposium on Lattice Field Theory

24-30 July 2016

University of Southampton, UK

\footnotetext{
${ }^{*}$ Speaker.
} 


\section{Introduction}

It is well known that the lattice local axial vector current $A_{\mu}^{\text {loc }}=\bar{\psi}(x) \gamma_{\mu} \gamma_{5} \psi(x)$ does not satisfy the continuum form of the axial Ward identity

$$
\partial_{\mu} A_{\mu}(x)=2 m P(x), \quad P(x)=\bar{\psi}(x) \gamma_{5} \psi(x),
$$

which is due to lattice artefacts. In most cases, where Wilson like fermions are used, the corresponding improved renormalized current

$$
A_{\mu}^{\mathrm{loc}, \overline{\mathrm{MS}}}(x)=Z_{A^{\mathrm{loc}}}\left(1+b_{A} a m\right)\left[A_{\mu}^{\mathrm{latt}, \mathrm{loc}}+a c_{A} \partial_{\mu} P^{\mathrm{latt}}\right]
$$

is taken to compute physical quantities, like the nucleon axial charge $g_{A}$. It turned out, however, that the resulting $g_{A}$ value are slightly below the experimental number and it requires a large effort to bring the lattice result into coincidence with it $[1,2,3,4,5]$. An alternative possibility is to use the point-split (ps) axial vector current

$$
A_{\mu}^{\mathrm{ps}}(x)=\frac{1}{2}\left[\bar{\psi}_{x} \gamma_{\mu} \gamma_{5} U_{\mu}(x) \psi_{x+a \hat{\mu}}+\bar{\psi}_{x+a \hat{\mu}} \gamma_{\mu} \gamma_{5} U_{\mu}^{\dagger}(x) \psi_{x}\right]
$$

which is known to fulfill the corresponding lattice axial Ward identity [6]. In [7] we could show that this identity is fulfilled both perturbatively (in one-loop) and nonperturbatively for the SLiNC action [8]. In this work we present implications of this nonlocal lattice form of the axial current the renormalization and the computation of the axial charge $g_{A}$.

\section{Renormalization}

It is known that the point-split lattice vector current $V_{\mu}^{\mathrm{ps}}(x)$ as obtained from the lattice vector Ward identity is conserved. This leads to the renormalization factor $Z_{V}^{\mathrm{ps}}=1$ whereas the nonconserved local counterpart differs by a finite number from that. One could speculate what behavior $Z_{A}^{\mathrm{ps}}$ computed for (1.3) shows. On the one hand $A_{\mu}^{\mathrm{ps}}(x)$ is also a result of the lattice axial vector Ward identity. On the other hand there appears an extra term which cannot be absorbed into a redefinition of the current. This is due to the fact that Wilson like actions break chiral symmetry. For the SLiNC action (where the fermionic part is a stout smeared version of a clover improved Wilson action) this extra term is a combination of the standard Wilson term and the clover term. We perform a nonperturbative calculation on a $32^{3} \times 64$ lattice at $\beta=5.50[a=0.074(2) \mathrm{fm}]$. In order to perform the chiral limit we use five $\kappa$ values along the flavor symmetric line $\left(\kappa_{l}=\kappa_{s}\right)$ corresponding to pion masses $M_{\pi}=470,440,400,340,290 \mathrm{MeV}$.

We use the nonperturbative RI'-MOM scheme [9] performing a linear chiral extrapolation for each $(a p)^{2}$ value. Afterwards we transform into the RGI and $\overline{\mathrm{MS}}$ schemes which coincide due to the lack of anomalous dimensions. The result is shown in Fig. 1. In the chosen momentum interval $Z_{A}$ is fitted as a linear function in $(a p)^{2}$. Variations of this interval determines the systematic error which dominates the fit error. We find $Z_{A}^{\mathrm{ps}}=1.0212(12)_{\mathrm{fit}}(47)_{\mathrm{sys}}$ which is very near to one. This is consistent with one-loop perturbative results using, however, a different gauge action [7]. That result would mean that practically quantities computed from this point-split axial vector current alone do not need to be renormalized. It remains to check, however, that this behavior remains valid for other $\beta$ values (lattice spacings). 


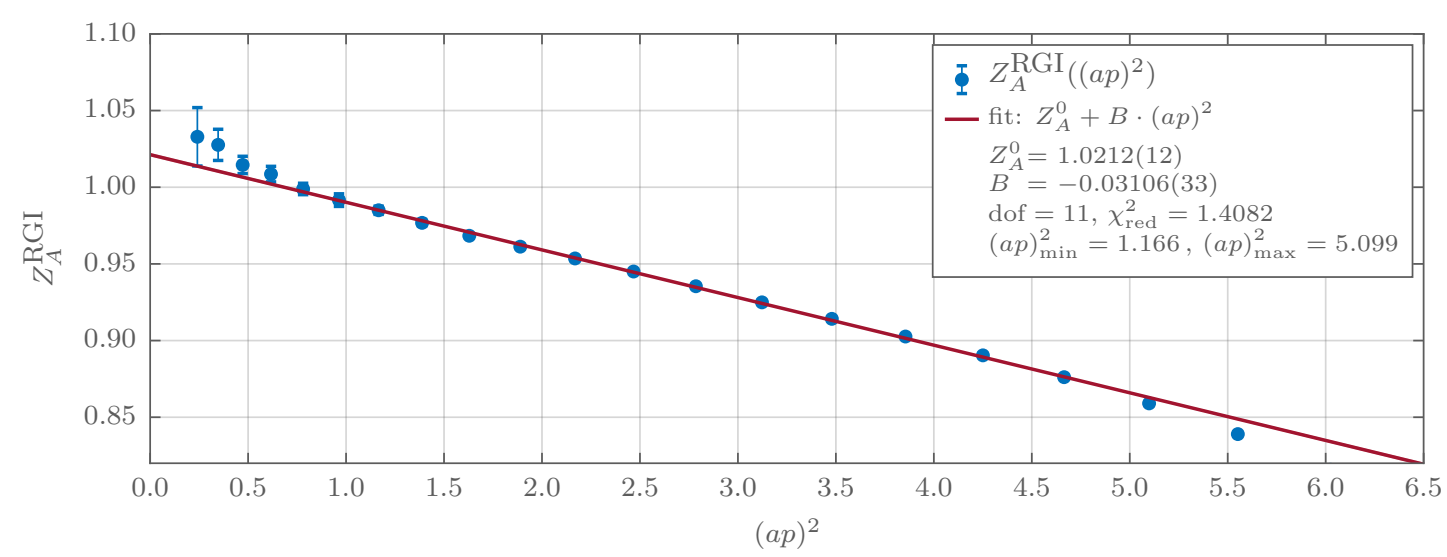

Figure 1: $Z_{A}^{\mathrm{RGI}}$ for $\beta=5.5$ as function of $(a p)^{2}$ for the point-split axial current $A=A^{\mathrm{ps}}$.

\section{Nucleon axial charge $g_{A}$}

The axial charge $g_{A}$ is an important quantity to understand the spin structure of the nucleon, but also plays a role in certain astrophysical processes. It can be measured in the $\beta$ decay of the neutron $\left(n \rightarrow p+e+\bar{v}_{e}\right)$ where it determines the angular distribution of the emitted electron. The current experimental value is given in [10] as $g_{A}=1.2723(23)$.

On the lattice $g_{A}$ is calculated from the forward matrix element of the axial vector current $A_{\mathrm{q}}^{\mu}$

$$
\left\langle\mathbf{p}, s\left|A_{\mathrm{u}-\mathrm{d}}^{\mu}\right| \mathbf{p}, s\right\rangle=2 g_{A} s^{\mu},
$$

where $|\mathbf{p}, s\rangle$ is a proton state with momentum $\mathbf{p}$ and spin $s^{\mu}$ and the inserted operator is $A_{\mathbf{u}-\mathrm{d}}^{\mu}=$ $A_{\mathrm{u}}^{\mu}-A_{\mathrm{d}}^{\mu}$. Being a nonsinglet quantity there are no contributions from disconnected quark lines. The relation (3.1) makes this observable to a benchmark test for lattice calculations. For a review of the current status see [5]. Despite the progress that has been made in the last years there remain a couple of challenges to be solved. Among them we mention the extrapolation to the physical point and the treatment of excited states.

In this work we take the point-split axial vector current (1.3) as the operator inserted in (3.1). As lattices we use $\left\{32^{3} \times 64, \beta=5.50[a=0.074(2) \mathrm{fm}], M_{\pi}=470,360,310 \mathrm{MeV}\right\}$ along the $\bar{m}=$ const. line and $\left\{48^{3} \times 96, \beta=5.80[a=0.059(3) \mathrm{fm}], M_{\pi}=427 \mathrm{MeV}\right\}$ at the flavor symmetric point. $g_{A}$ has to be estimated from the ratio of the 3-point function to the 2-point function

$$
R\left(t_{i}, t_{f}, \tau\right)=\frac{G_{3}\left(t_{i}, t_{f}, \tau\right)}{G_{2}\left(t_{i}, t_{f}\right)} \rightarrow g_{A}
$$

with $\left(t_{f}-t_{i}\right)$ - the source-sink distance, $\tau$ - the source-operator insertion distance. It is clear that a meaningful determination of $g_{A}$ is possible only if the ratio exhibits a pronounced plateau, ideally independent on $\left(t_{f}-t_{i}\right)$ and $\tau$.

One of the main challenges of this kind of computations is the handling of excited states. There are various techniques which are used to take them into account. Among them we have the summation, the multi-exponential fit and the variational methods [11, 12, 13, 14].

It turns out that the source-sink distance determines the form and the height of the plateau of the ratio $R\left(t_{i}, t_{f}, \tau\right)$ defined in (3.2) (see Fig. 2). This is directly connected to the influence of 


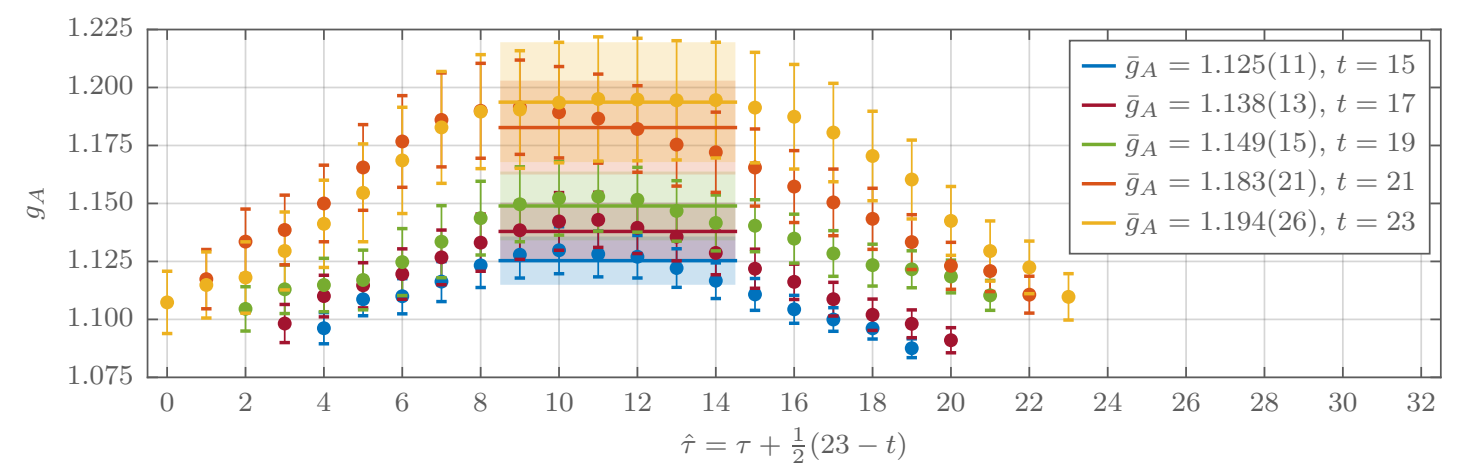

Figure 2: Plateaus of $R\left(t_{i}, t_{f}, \tau\right)$ for various $t_{\text {sep }}=\left(t_{f}-t_{i}\right)$ as function of operator insertion time $\tau$ for $\beta=5.80$.

excited states which diminish the height for smaller separations. Investigations in [14] suggest that the variational method shows very stable results.

In our computation, however, we used the 3 -exponential fit method which includes the first three energy levels $\left(t=t_{\text {sep }}\right)$. The fit to the ratio (3.2) has the form

$$
\begin{aligned}
F_{g_{A}}(t, \tau)=g_{A}^{\mathrm{fit}} & {\left[1+C_{10}\left(e^{-\tau M_{10}}+e^{-(t-\tau) M_{10}}\right)+C_{11} e^{-t M_{10}}\right.} \\
& +C_{20}\left(e^{-\tau M_{20}}+e^{-(t-\tau) M_{20}}\right)+C_{22} e^{-t M_{20}} \\
& \left.+C_{21}\left(e^{-\tau M_{21}} e^{-t M_{10}}\right)+C_{21}\left(e^{-t M_{20}} e^{-\tau M_{21}}\right)\right] \\
& \times\left[1+D_{10} e^{-t M_{10}}+D_{20} e^{-t M_{20}}\right]^{-1},
\end{aligned}
$$

where $M_{i k}=M_{i}-M_{k}$ and the $M_{i}$ are the masses of the ground state $(i=0)$ and the next two excited states $(i=1,2)$. They can be determined rather precisely from the corresponding 2-point functions, as shown in Fig. 3. The fit (3.3) is performed for the parameters $g_{A}^{\mathrm{fit}}, C$ and $D$ over the available data sets $(t, \tau)$ simultaneously. An example is shown in the right of Fig. 3. where we fit over the whole data set with all available separations $t_{\text {sep }}=15 \ldots 23$.

Our final results for $g_{A}$ at $\beta=5.50\left(M_{\pi}=470 \mathrm{MeV}\right)$ and $\beta=5.80$ are shown in Fig. 4 . We compare them for $\beta=5.50$ with the variational method [14] which has been obtained for the local axial curent on the same lattice. It is obvious that the plateau values depend very much on the source-sink separations. The 3-exponential fit gives a higher value towards the experimental result. It can be recognized that (using the same lattice parameters) the axial charge for the point-split current is nearer to the experimental value than the value using the local current. This should be seen also in connection to the corresponding $Z$ factor $\left(Z_{A}^{\mathrm{ps}}=1.0212\right)$ which indicates that we are rather close to the continuum. Furthermore, we did not find a significant dependence on the three pion masses at $\beta=5.50$. For $\beta=5.80$ the final fit $g_{A}^{\text {fit }}$ is even larger. This shows a tendency to increase $g_{A}(\beta)$ with increasing $\beta$ (decreasing lattice spacing $a$ ) which is encouraging. However, for a sound comparison with experiment it remains to perform a careful extrapolation to the physical point. 

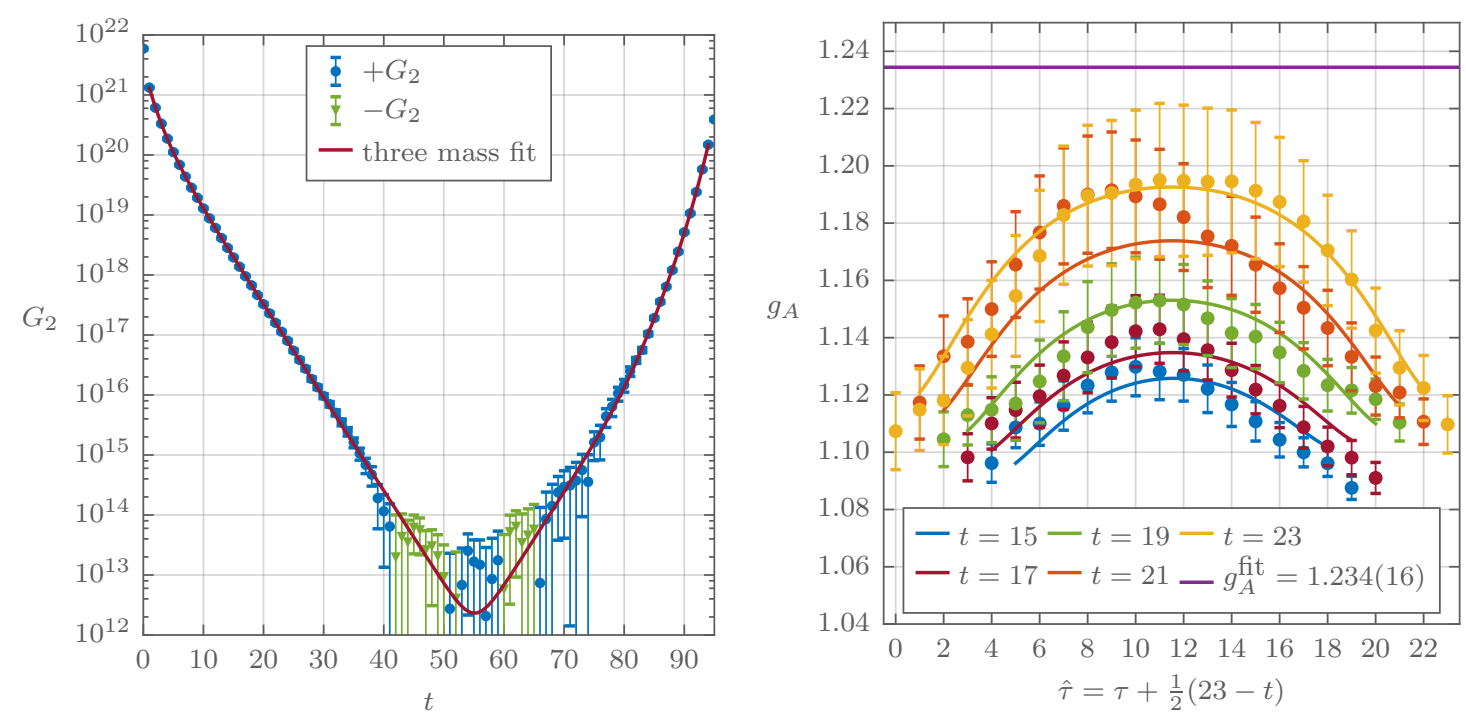

Figure 3: Left: Three mass fit to the 2-point correlation function for $\beta=5.80$. Right: Result of the global fit (3.3) for $\beta=5.80$.

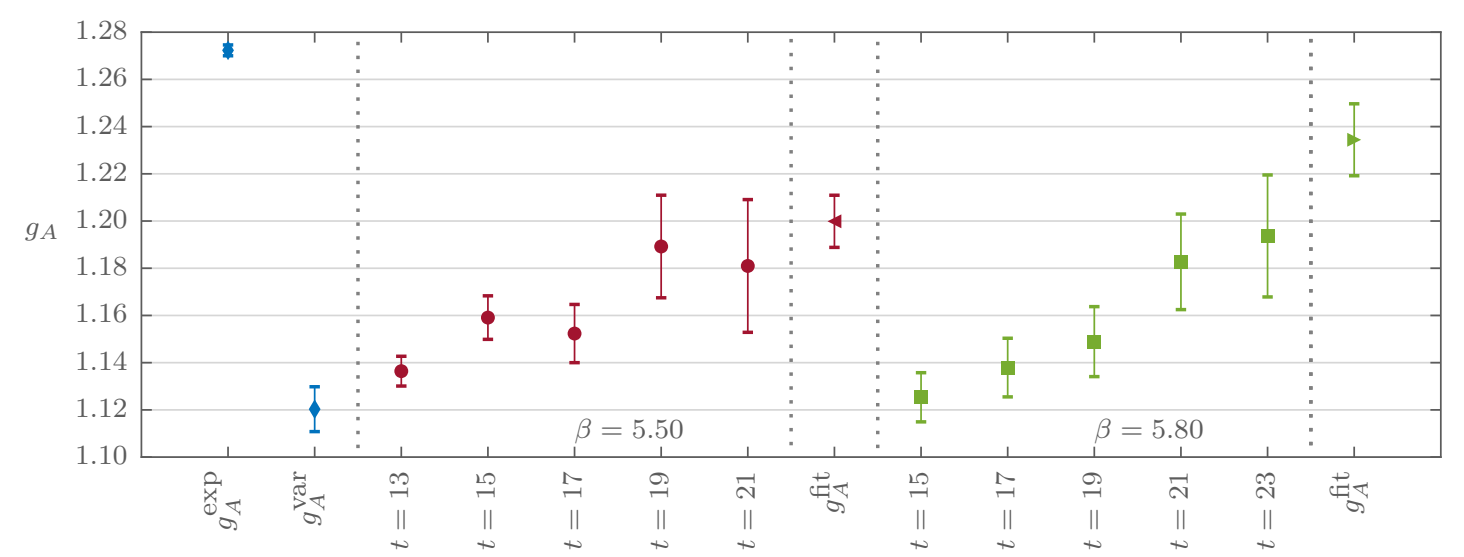

Figure 4: $g_{A}$ values using different methods at the flavor symmetric points. $\quad \beta=5.50$ and $M_{\pi}=$ $470 \mathrm{MeV}: g_{A}^{\mathrm{var}}$ : local axial current (variational method), point-split axial current (plateau values at $t=$ $t_{\text {sep }}=13,15,17,19,21$ ) and 3-exponential fit $g_{A}^{\text {fit }} \beta=5.80$ : point-split axial current (plateau values at $\left.t=t_{\text {sep }}=15,17,19,21,23\right)$ and 3-exponential fit $g_{A}^{\text {fit }}$. The experimental value is denoted as $g_{A}^{\text {exp }}$.

\section{Acknowledgements}

The numerical configuration generation (using the BQCD lattice QCD program [15]) was carried out on the IBM BlueGene/Q using DIRAC 2 resources (EPCC, Edinburgh, UK) and the BlueGene/P and Q at NIC (Jülich, Germany). The data analysis (using the Chroma software library [16]) was performed on the SGI ICE 8200 and Cray XC30 at the HLRN (The North-German Supercomputer Alliance). HP was supported by the DFG grant SCHI 422/10-1. PELR was supported in part by the STFC under contract ST/G00062X/1 and JMZ by the Australien Research Council Grant No. FT100100005 and DP140103067. We thank all funding agencies. 


\section{References}

[1] R. Horsley, Y. Nakamura, A. Nobile, P. E. L. Rakow, G. Schierholz and J. M. Zanotti, Phys. Lett. B 732 (2014) 41 [arXiv:1302.2233 [hep-lat]].

[2] G. S. Bali et al., Phys. Rev. D 91 (2015) no.5, 054501 [arXiv:1412.7336 [hep-lat]].

[3] C. Alexandrou, K. Hadjiyiannakou and C. Kallidonis, Phys. Rev. D 94 (2016) no.3, 034502 doi:10.1103/PhysRevD.94.034502 [arXiv:1606.01650 [hep-lat]].

[4] T. Bhattacharya, V. Cirigliano, S. Cohen, R. Gupta, H. W. Lin and B. Yoon, arXiv:1606.07049 [hep-lat].

[5] S. Collins, plenary talk given at the conference Lattice 2016.

[6] M. Bochicchio, L. Maiani, G. Martinelli, G. C. Rossi and M. Testa, Nucl. Phys. B 262 (1985) 331.

[7] R. Horsley, Y. Nakamura, H. Perlt, P. E. L. Rakow, G. Schierholz, A. Schiller and J. M. Zanotti, PoS LATTICE 2015 (2016) 138 [arXiv:1511.05304 [hep-lat]].

[8] N. Cundy et al. [QCDSF Collaboration], Phys. Rev. D79 (2009) 094507 [arXiv:0901.3302 [hep-lat]].

[9] G. Martinelli, C. Pittori, C. T. Sachrajda, M. Testa and A. Vladikas, Nucl. Phys. B 445 (1995) 81 [hep-lat/9411010].

[10] K. A. Olive et al. [Particle Data Group Collaboration], Chin. Phys. C 38 (2014) 090001.

[11] S. Capitani, M. Della Morte, G. von Hippel, B. Jäger, A. Jüttner, B. Knippschild, H. B. Meyer and H. Wittig, Phys. Rev. D 86 (2012) 074502 [arXiv:1205.0180 [hep-lat]].

[12] B. J. Owen, J. Dragos, W. Kamleh, D. B. Leinweber, M. S. Mahbub, B. J. Menadue and J. M. Zanotti, Phys. Lett. B 723 (2013) 217 [arXiv:1212.4668 [hep-lat]].

[13] B. Yoon et al., Phys. Rev. D 93 (2016) no.11, 114506 [arXiv:1602.07737 [hep-lat]].

[14] J. Dragos et al., arXiv:1606.03195 [hep-lat].

[15] Y. Nakamura and H. Stüben, PoS LATTICE 2010 (2010) 040 [arXiv:1011.0199 [hep-lat]].

[16] R. G. Edwards et al. [SciDAC and LHPC and UKQCD Collaborations], Nucl. Phys. Proc. Suppl. 140 (2005) 832 doi:10.1016/j.nuclphysbps.2004.11.254 [hep-lat/0409003]. 